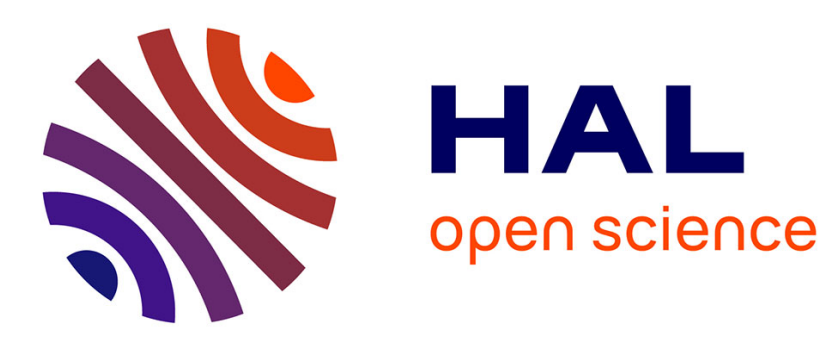

\title{
Online adaptation of the IEEE 802.15.4 parameters for wireless networked control systems
}

\author{
Najet Boughanmi, Ye-Qiong Song, Eric Rondeau
}

\section{To cite this version:}

Najet Boughanmi, Ye-Qiong Song, Eric Rondeau. Online adaptation of the IEEE 802.15.4 parameters for wireless networked control systems. 8th IFAC International Conference on Fieldbuses and networks in industrial and embedded systems, FET'2009, May 2009, Ansan, South Korea. pp.CDROM. inria00430957

\section{HAL Id: inria-00430957 https://hal.inria.fr/inria-00430957}

Submitted on 10 Nov 2009

HAL is a multi-disciplinary open access archive for the deposit and dissemination of scientific research documents, whether they are published or not. The documents may come from teaching and research institutions in France or abroad, or from public or private research centers.
L'archive ouverte pluridisciplinaire HAL, est destinée au dépôt et à la diffusion de documents scientifiques de niveau recherche, publiés ou non, émanant des établissements d'enseignement et de recherche français ou étrangers, des laboratoires publics ou privés. 


\title{
Online adaptation of the IEEE 802.15.4 parameters for wireless networked control systems
}

\author{
N. Boughanmi ${ }^{*}$ YQ. Song ${ }^{* *}$ E. Rondeau ${ }^{* * *}$ \\ * LORIA-CRAN-INPL boughanm@loria.fr \\ ** LORIA-INPL 615 rue du Jardin botanique-CS 20101 \\ 54603 Villers-lès-Nancy Cedex France song@loria.fr \\ *** CRAN Université Henri Poincaré \\ Vandoeuvre-lès-Nancy Cedex France eric.rondeau@cran.uhp-nancy.fr
}

\begin{abstract}
:
The IEEE 802.15.4 has been studied for its possible use to support networked control systems. A careful management of the network resources allows the enhancement of the quality of control (QoC) of the controlled system. This can be achieved by a co-design approach to manage the interaction between the WNCS and the wireless network in order to online adapt the QoC. This approach allows control loops to have access to the communication medium according to the state of the controlled system meanwhile attempting to optimize the overall control performance. When the QoC of the controlled system is not sufficient, the quality of service of the network is adapted online. This adaptation is realized by modifying the macMinBE parameter of the MAC protocol of the IEEE 802.15.4. Moreover, an implementation way of this scheme using the IEEE 802.15.4 protocol is presented. Simulation results show that this approach improves the control performance.
\end{abstract}

\section{INTRODUCTION}

Networked control systems (NCS) [12] have an increasing number of applications in military, industrial, medical and commercial fields. Since wired networks cause significant limitations in terms of mobility, flexibility and extensibility, wireless networks are an effective alternative to overcome these problems. Hence, Wireless Networked Control Systems (WNCS) are getting a strong interest from both academic and industrial communities. The IEEE 802.15.4/ZigBee is an interesting standard for some WNCS requiring low cost and low energy consumption (e.g., the case of the RUNES project $[\mathrm{RUN}]$ ). Moreover industrial WirelessHART [1] uses the IEEE 802.15.4-based Physical layer and MAC PDU.

In WNCS, the quality of control (QoC), i.e., the performance $[4,2]$ delivered by each closed-loop operation depends not only on the controller design but also on the quality of service (QoS) offered by the wireless network. The degradation of the QoC of the controlled process can be caused by the network or by the controlled system. In order to manage this QoC many researchers try to enhance the QoS offered to the WNCS. Thus several network resource allocation techniques for WNCS have been proposed. These techniques are based on static strategies that ensure average control performance at the expenses of permanently occupy the available bandwidth. An adaptive online QoC management protocol is proposed: if the QoC of the controlled process is not sufficient, the network offers more resources to the WNCS. Moreover, since the

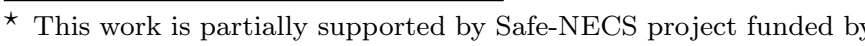
ANR under ANR-05-SSIA-0015-03. degradation of the QoC can be due to the control loop, if there is no enhancement of the QoC for a certain amount of time, action must be taken on the control loop ( changing the sampling period for example). This work deals with the degradation of the QoC caused by the lack of network resources.

The adaptation of the QoS is ensured through a priority mechanism which adapts the backoff exponent value in the IEEE 802.15.4 MAC. Two priority mechanisms are studied: a probabilistic one and a deterministic one. Then, an implementation of this protocol using the IEEE 802.15.4 is proposed. To illustrate its benefit, this approach is implemented in TrueTime [9], a Matlab/Simulink simulator, and experimented on a simulated cart. The simulation results reveal that this adaptive online QoC management protocol enhances the QoC of the controlled system.

The rest of the paper is organized as follows. The related work is presented in Section 2. Section 3 deals with the considered WNCS. In Section 4, an overview of IEEE 802.15.4 is presented. In Section 5, the limitations of the CSMA/CA mechanism are shown. Thus, in Section 6 an online adaptive protocol is proposed. Then, in Section 7 , an implementation of this protocol over IEEE 802.15.4 is proposed. In Section 8, the simulation results are presented. Section 9 gives the conclusion and the future work.

\section{RELATED WORK}

There are several works which deal with the resource allocation for control loops. Marti et al. [7] studied the CPU resource management and showed that by using feedback to dynamically allocate resources to controllers as 
a function of the current state of their controlled systems, control performance can be significantly improved. They present an optimal resource allocation policy that maximizes control performance within the available resources. In this work the management is dependent of the controller since when there is a perturbation, the sampling period is adapted.

Velasco et al. [11] propose a dynamic approach to bandwidth management in networked control systems that allows control loops to consume bandwidth according to the dynamics of the controlled process meanwhile attempting to optimize overall control performance. This is done by augmenting the original state-space representation of each controlled system with a new state variable that describes the network dynamics.

Ji et al. [5] assign the network-bandwidth dynamically to each control loop according to the quality of performance of each control loop. This is done by using an adaptive controller.

All these works try to adapt the control loop to its environment. Thus, the parameters of the control loop are changed, especially the sampling period.

Marti et al. [8] propose an approach to adaptive controllers for NCS that online adapts the control decisions according to the dynamics of both the application and the executing platform. This approach offers capabilities for dynamic management of QoC through message scheduling. They formulate a scheduling strategy that uses feedback information from the control application in order to schedule messages in such a way that the degrading effects of the message latencies are minimized. Thus, the overall QoC is improved. However, this strategy was not analyzed nor tested.

All above works are designed for wired networks. For the best of our knowledge, there is no QoC online adaptive strategy for WNCS using the IEEE 802.15.4 which adapts the QoS of the network to the control loop requirements. This QoS management can be done through the service differentiation. Koubâa et al. [6] proposed a simple differentiated service scheme for slotted CSMA/CA in IEEE 802.15.4 to improve the performance of time sensitive message. In [10], the authors have modified the initial value of backoff exponent in IEEE 802.15.4 MAC (slotted CSMA/CA) and proposed an adaptive backoff exponent (ABE) algorithm. These works only focused on the slotted CSMA/CA and they are not linked to networked control systems. In this paper, an online adaptive scheme is proposed for wireless networked control systems.

\section{THE CONSIDERED WIRELESS NETWORKED CONTROL SYSTEM}

A system of a cart whose movement is guided along a rail [4] is considered as an example. We aim to control the cart's position. The variable characterizing the system's state $\left(x^{T}=[d \dot{d}]\right)$ are: $d$, the cart position along the rail measured from a reference point, and, $\dot{d}$ its velocity. The simplified model of the system is described by the following differential equation where $u$ is the input and $k_{1}, k_{2}$ two parameters :

$$
\ddot{d}=-k_{1} \dot{d}+k_{2} u
$$

where $k_{1}=12.6559$ and $k_{2}=1.9243$ according to the system identification. The continuous model of the system based on the state space representation is given by:

$$
\left\{\begin{array}{l}
\dot{x}=A x+B u \\
y=C x+D u
\end{array}\right.
$$

where

$$
A=\left[\begin{array}{cc}
0 & 1 \\
0 & -k_{1}
\end{array}\right], B=\left[\begin{array}{c}
0 \\
k_{2}
\end{array}\right]
$$

The output of the system corresponds to its state variable $(y=x)$. So

$$
C=\left[\begin{array}{ll}
1 & 0 \\
0 & 1
\end{array}\right], D=0 .
$$

The used controller is a State Feedback Controller defined by

$$
u_{t}=L\left(x_{r e f}-x_{t}\right)
$$

where $x_{r e f}=\left[\begin{array}{ll}r & 0\end{array}\right]^{T}, r$ is the reference position of the cart and $L=\left[\begin{array}{ll}k_{c} & k_{d}\end{array}\right]$. The parameters $k_{c}$ and $k_{d}$ were evaluated by applying the LQR method. For a sampling period equal to $0.01 s, k_{c}=121$ and $k_{d}=6.5$ [4]. A wireless network containing the sensor $(\mathrm{S})$, the controller (C) and the actuator (A) is introduced. The network adds new delays: $\tau^{s c}$ is the delay between the sensor and the controller, and $\tau^{c a}$ is the delay between the controller and the actuator as shown in Figure 1.

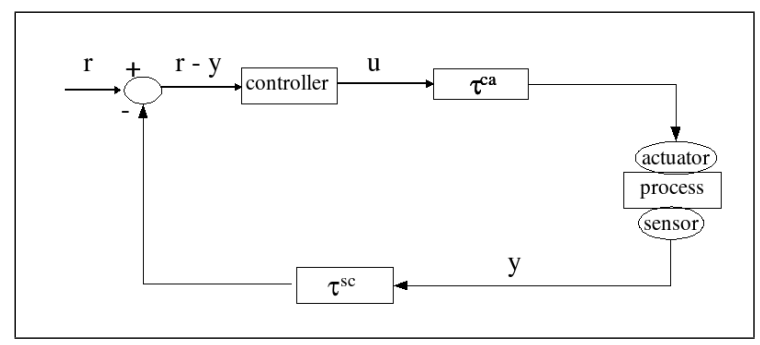

Fig. 1. The networked control system architecture

The maximum value of the sampling period is $100 \mathrm{~ms}$ for keeping the system stable. This paper provides a study of the impact of the network performance on the controlled system in a realistic case. Besides, the QoC is managed online by adapting the QoS offered to the controlled cart.

\section{IEEE 802.15.4 PROTOCOL OVERVIEW}

The MAC protocol supports two operational modes that may be selected by the coordinator: beacon-enabled mode and non beacon-enabled mode.

- Non beacon-enabled mode : Medium access control is provided by an unslotted CSMA/CA (Carrier Sense Multiple Access/ Collision Avoidance) mechanism (using a random backoff time based on backoff period and Backoff Exponent (BE). There is no priority mechanism so no way to isolate a particular flow among all traffic. 
- Beacon-enabled mode : Beacons are periodically sent by Zigbee coordinator to synchronize nodes that are associated with it, and to identify the Personal Area Network (PAN). Superframe is contained between two consecutive beacon frames. The superframe structure is used to manage communication between these devices. The superframe contains a Contention-Access Period (CAP) and ContentionFree Period (CFP), and it may include an inactive period.

\section{CSMA/CA}

In a realistic case, there are other nodes than the control loop nodes using the wireless network. In our example, the communication channel is shared by 2 cyclops (sensors equipped with camera) and a main control unit as shown in Figure 2. Hence, the wireless network is used to transmit image packets from cyclops to the main control unit. The image sensor has CIF resolution $(352 \times 288)$. Each cyclops sends, periodically, 133 bytes. In the following, the plant behavior is studied for different perturbation load. The transmission period is modified in order to see the effect of the new additional network load on the WNCS. The QoC of the controlled cart is satisfying if the additional network load is less than $44 \%$ of the total bandwidth. However, if this additional network load reaches $44 \%$ (i.e. the transmission period of each cyclops $=0.02 \mathrm{~s})$, the cart becomes unstable. Thus, the additional load of $44 \%$ of the total bandwidth is the upper limit for the stability of our WNCS. In general, the unslotted CSMA/CA of the non beacon-enabled mode is not convenient for WNCSs since influenced by the total network load.

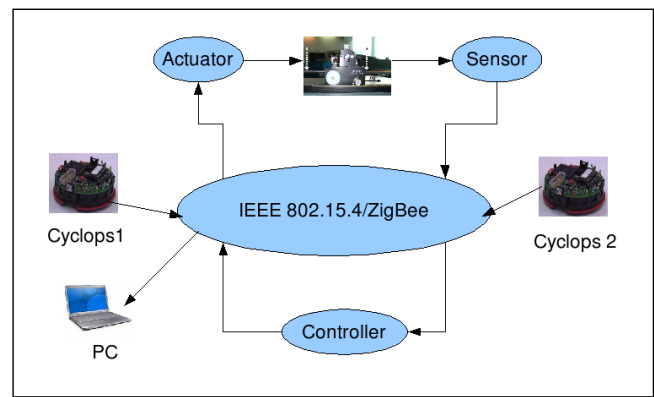

Fig. 2. IEEE 802.15.4/Zigbee using CSMA/CA: shared network

\section{ONLINE ADAPTIVE PROTOCOL}

A one-hop wireless network with peer-to-peer topology is considered. Moreover, the MAC protocol operational mode is the beacon-enabled one and we are interested in the Contention Access Period of the superframe. To regulate the QoS offered to the WNCS, the prioritized CSMA/CA mechanism ( explained in subsection 6.3) is used instead of the Guaranteed Time Slot (GTS) one. GTSs may be allocated by the network coordinator to applications with real-time and specific data bandwidth requirements. In fact, this scheme offers an alternative choice to GTS one. For hard real-time, GTS is preferable while for soft real-time, prioritized CSMA/CA, that will be described below, could be suitable. In addition, this will overcome the problems of GTS. In fact, the number of the control loop having the same sampling period is limited as shown in [3] since at most only 7 GTS slots can be used. Moreover, the GTS deallocation is not easy and that makes the GTS mechanism rigid.

In order to provide the required QoC to the control loop to maintain the stability of the controlled process, the required QoS must be guaranteed to the WNCS. Thus, a joined design algorithm is proposed: the QoS is adapted depending on the QoC.

A dynamic QoC management is proposed. This is ensured by an additional "network control loop" presented in Figure 8 and will be detailed in Section 7. Here, the principle is stated: depending on the current state of the QoC metric, the given QoS to the WNCS can be adapted online. Thus, the QoC of the plant is improved.

It is supposed that there is no sampling period change because it may cause the plant instability and this paper deals only with the problems caused by the network.

\subsection{QoC metric}

The QoC of the WNCS is evaluated using the controlled process error $e$. If the WNCS is stable, the error is bounded. Thus, the same criterion, as in [5], is adopted: the error should be bounded by a threshold to ensure the required $\mathrm{QoC}$ to the plant. This threshold depends on the controlled process and on the reference value if there is any. If $e>$ threshold, the WNCS is considered to be in a critical situation and action has to be taken. The trouble is caused either by the controlled system itself or by the network (overloaded network). Action should be taken on the network for a certain period of time through offering more resources to the WNCS. If the situation is not improved (there is a problem in the control loop), action should be taken on the WNCS by changing the sampling period for example. This work deals with the adaptation of the QoS in order to enhance the QoC.

For the systems with architecture presented in Figure 1, the error $e$ is equal to $|r-y|$ where $r$ is the reference and $y$ is the process response. In order to have a good QoC, the condition is

$$
|r-y|<\text { threshold }+r
$$

has to be satisfied. The reference value is added in order to take into account the case where there is a change in the reference value that makes $|r-y|=r$ and there is no network problem.

\subsection{Dynamic management of the QoC}

The QoC of the controlled process is dynamically managed through the QoC metric $e$ (the system error). First, the controller checks the error value so that it can decide its priority level because there are two: the maximum priority, and the normal one. If the error $e$ is higher than the threshold, then the WNCS is in critical situation. Thus, the controller priority is set to its maximum value. Else, the controller has a normal priority. This priority data is expressed through the random range of the waiting delay of the CSMA/CA. There are two alternatives : 
(1) this range is set to a big one for all the nodes in the network, then when the controller priority is equal to the maximum value, this range is decreased for both the sensor and the controller,

(2) this range is set to the default one for all the nodes, then if the controller priority is equal to the maximum value, this range is increased for all the nodes in the network except the sensor and the controller.

The first solution is the most suitable for hard real-time applications considering the robustness aspect. However, it induces the waste of the network resources by the large waiting delay. Thus, the second solution is adopted.

The priority parameter is transferred to the MAC layer which will send it to the WSN coordinator. This coordinator is in charge of informing all the other nodes of the current controller's priority. Once one node gets the controller priority information, it decides if it will apply the CSMA/CA either with the probabilistic priority or without. This decisions is related to the controller priority value. Moreover, the transition between the two mechanisms is done progressively. In fact, if the controller priority is equal to the maximum value, the range of the random delay is increased, else, it decreased until it is equal to the default range.

\subsection{QoS adaptation: CSMA/CA with priority}

The CSMA/CA mechanism for IEEE 802.15.4 uses the random waiting delay for collision avoidance. It uses the backoff exponent (BE) which is related to how many backoff period (BP) a device must wait before attempting to assess the channel activity. The algorithm attempts to avoid collision by waiting during a given delay randomly generated in the range of $\left[0,2^{B E}-1\right] \times B P$. If batterylife extension is activated then, $B E=\min (2, \operatorname{macMin} B E)$ else, $B E=\operatorname{macMinBE}$ where macMinBE attribute specifies the minimum of backoff exponent, which is set to 3 by default. This aspect is exploited in order to differentiate the services offered by the WSN. We propose to vary the randomly generated waiting delay depending on the priority of the packet. Thus, by choosing a higher macMinBE for the applications different from the control loop, the probability to have a longer delay is increased and the control loop nodes will be able to send their data packets.

The nodes are divided into two classes: the high priority (h) class contains the nodes of the WNCS (sensor, controller, and actuator), and the low priority (l) class is composed of the other nodes present in the WSN. $B E_{C L}$ represents the $B E$ of the control loop nodes macMinB $E_{C L}$ its $\operatorname{macMin} B E$, and it is set to the default value 3 . The macMinBE $E_{O A}$ and $B E_{O A}$ are the macMinBE and the $B E$ of the other applications sharing the WSN. When the batterylife extension is not activated, the control loop nodes have to wait random $\left[0,2^{B E_{C L}}-1\right] \times B P($ random $[0,7] \times B P)$.

Probabilistic priority The macMinB $E_{O A}$ of the low priority nodes is increased in order to make their data packets wait during a delay randomly generated in a longer range $\left(\right.$ random $\left.\left[0,2^{\text {macMinB }} E_{O A}-1\right] \times B P\right)$ than the one for high priority nodes. Figure 3 shows these two ranges
.Thus, a probabilistic priority is assigned to the nodes in the WSN by modifying the range for the random delay. Hence, the higher priority nodes (the control loop nodes here) have more chance to send their data packets.

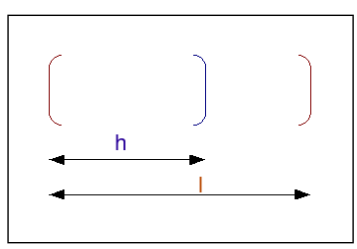

Fig. 3. Waiting ranges for high and low priority nodes using the probabilistic priority

Figure 4 shows the $\operatorname{macMin} B E_{O A}$, obtained by simulations, which allows the control loop to be stable for different loads. For a small load (less than 35\%), there is no need to increase the macMinBE since the default value 3 can afford the stability for the WNCS. When the disturbing load becomes important, the control loop becomes to be unstable because of the long delay and the packets losses. Thus, increasing the macMinBE $E_{O A}$ allows the control loop to reach the stability in spite of the perturbing load. For the considered control loop, the value of 7 for $\operatorname{macMinBE}$ affords the stability for the control loop for a perturbation load of $90 \%$ of the total bandwidth.

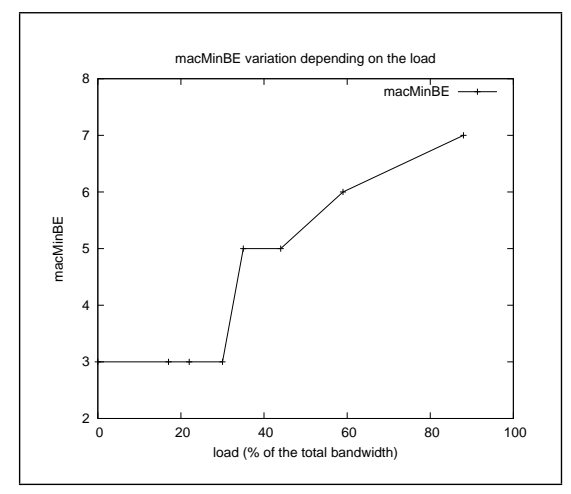

Fig. 4. $\operatorname{macMinB} E_{O A}$ variation depending on the perturbation load

Collisions still exist since the nodes of WSN can choose the same waiting delay.

Deterministic priority This part deals with the minimization of the collisions between nodes of the two classes (control loop and other applications). Thus, the intersection between the waiting ranges of the high and low priority nodes is eliminated as shown in Figure 5. The lowerpriority applications will wait during a delay randomly generated in the range of $\left[\right.$ variable, $2^{B E_{O A}}-1$ ] backoff periods. The question is how to choose the variable's value. This variable is set to $2^{B E_{C L}}$ so that there is no collision between the members of the two classes (variable $=8$ ).

Figure 6 presents the evolution of the macMinBE $E_{O A}$ which allow the WNCS to be stable for different perturbation loads. The macMinBE $E_{O A}$ value, obtained using this method, is less than the one found when only the macMinBE is modified. Thus, separating the ranges of random waiting value of the two classes improves the QoS 


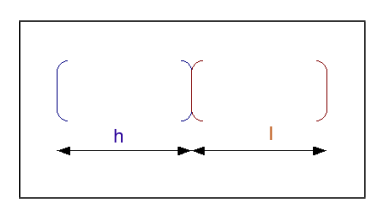

Fig. 5. Waiting ranges for high and low priority nodes using the deterministic priority

offered to the WNCS. In the following of this work, the deterministic priority scheme is adopted.

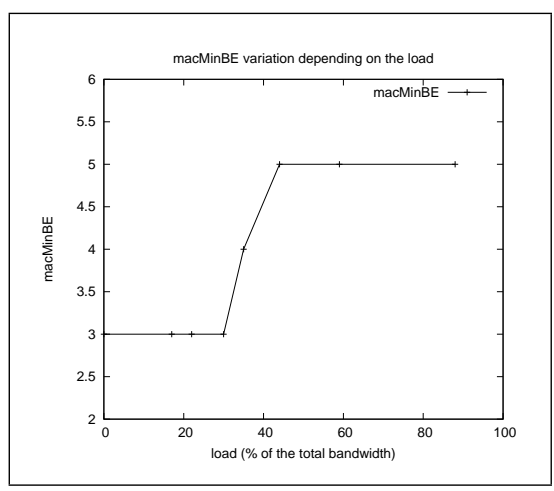

Fig. 6. macMinB $E_{O A}$ variation depending on the perturbation load

Online probabilistic priority adaptation Action will be taken on both the variable and macMinBE $E_{O A}$. Moreover, since a static assignment of these variables can lead to the under-use of the network resources due to the large waiting delay, these variables are adapted online depending on the controller's priority level. Thus, if the control loop is in a critical situation, the variable is set to 8 and the $\operatorname{macMinB} E_{O A}$ is increased by 1 in order to enlarge the waiting delay, else the variable is set to 0 and the $\operatorname{macMinB} E_{O A}$ is decreased by 1 .

\subsection{How to choose the threshold?}

The QoS management depends on the QoC metric which is represented by the controlled system error. Thus, the upper bound of the allowed error ( $e \leq$ threshold), the threshold, has to be chosen carefully. This threshold is defined, in equation 5, by the control loop threshold (threshold trocess $\left._{\text {) }}\right)$ and a security_margin.

threshold $=$ threshold ${ }_{\text {process }}-$ security_margin

Then, the delay introduced by the network should be taken into account in order to make the new QoS effective. This delay represents the propagation time of the new priority value to all the nodes. This time is composed of the transmission time of the priority message from the controller to the coordinator and the transmission time of the beacon as shown in Figure 7.

The network delay $d_{n e t}$ is given by

$$
d_{\text {net }}=\text { random backof } f+\text { waiting time. }
$$

In the beacon-enabled mode, the beacons are sent periodically (else if they are event-triggered this will cause an additional network load). Moreover, the random backof $f$ $(R B)$ value will be set to its maximum to get the worst case value $\left(R B=\operatorname{random}\left[0,2^{B E}-1\right] * B P\right)$. The needed

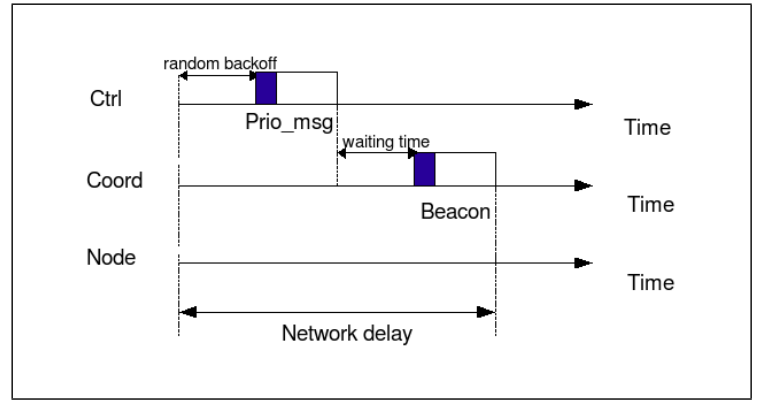

Fig. 7. The network delay to activate the probabilistic priority mechanism

time to send the beacon will vary depending on the current time position in the beacon interval. The waiting time is set to the value of the Beacon Interval BI (the worst case). Thus, the equation 6 is equivalent to

$$
d_{\text {net }}=\max (R B)+\min (B I) .
$$

The maximum $\mathrm{RB}$ is calculated as $\max (R B)=7 * B P$ where $\mathrm{BP}$ is equal to $320 \mu \mathrm{s}$. The beacon interval is defined as follow

$$
B I=\text { aBaseSuperframeDuration } \cdot 2^{B O}
$$

where $0 \leq B O \leq 14$ and $B O$ is the beacon order. The minimum value of $B I$ is calculated with $B O$ equal to 0 and $\min (B I)$ is equal to $15.36 \mathrm{~ms}$. Thus, the network delay is calculated as

$$
d_{\text {net }}=\max (R B)+\text { aBaseSuperframeDuration } \cdot 2^{B O} \text {, }
$$

when $B O=0, d_{n e t}=17.6 \mathrm{~ms}$.

Relation between the $d_{n e t}$ and $T_{e}$ The new priority information (or update) has to arrive on time to the network's nodes to be taken into account. Each new priority update has to be effective before a new one arrives. Since the controller is event triggered by the sensor which is periodical with the sampling period $T_{e}$, the minimum interval between two control message is equal to $T_{e}$. Thus, the constraint to satisfy, so that the enhancement of the QoS offered to the considered control loop is achieved, is the following: $T_{e} \geq d_{n e t}$.

In order to use the probabilistic priority mechanism, the minimal sampling period $T_{e_{\text {min }}}$ of the control loop has to be as the following: $T_{e_{m i n}}=d_{n e t}$ so $T_{e_{m i n}}=17.6 \mathrm{~ms}$.

\section{IMPLEMENTATION CONSIDERATION}

The proposed protocol takes into account the QoC of the control loop in order to regulate the QoS offered by the network to the WNCS. This protocol is divided in three actors: the controller of the considered control loop, the WSN coordinator, and the nodes which do not belong to this control loop. Figure 8 presents the network control loop with the different actors and message changes and details are given in the following parts. 


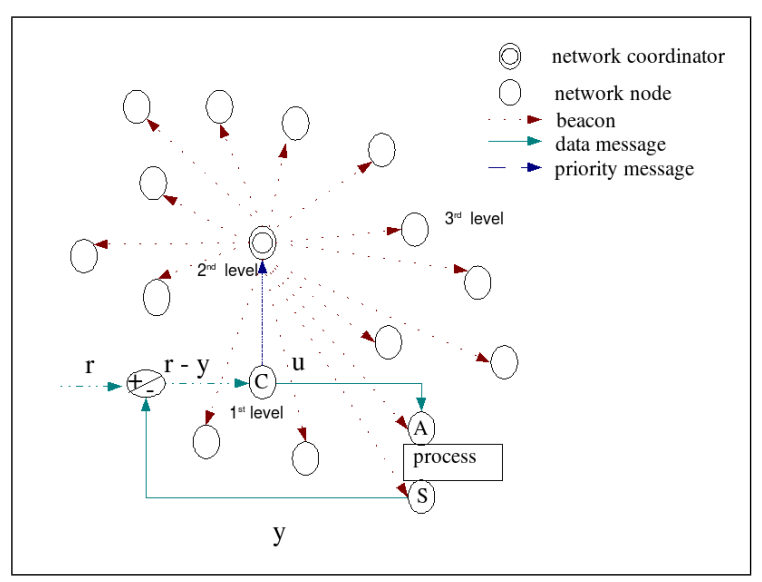

Fig. 8. Control over network (C: controller, A: actuator, and S: sensor)

\subsection{First level: the controller $i$}

At the application layer, the controller check the error value $e$. The control loop can tolerate the error if it is less than a threshold which allows to ensure the required QoC. Depending on the error value, the priority of the controller will be set. If $e>$ threshold then the priority will be equal to the maximum value max_prio, else, the priority will be set to the normal priority value normal_priority. The priority information will be transferred to the MAC layer through the payload added to the data part of the message to send to the actuator.

When the MAC layer gets the message to send to the actuator from the upper layer, it takes the priority value contained in the frame payload. This value will be converted to a boolean value and will be sent in a message to the WSN coordinator. Then, the MAC layer will send the first message to the actuator.

The question, now, is: how to indicate that the message is with priority one? There are two possible solutions :

(1) use a MAC command frame and indicate that it is a priority message by setting the command frame identifier to $0 \times 00$ since this value is not used by the standard. The first bit of the command payload will be set to the prio_msg_data.

(2) use the first bit of the MAC payload to indicate that this message deals with the priority. Thus if this bit is equal to 1 so the message is a priority one, and the following bit is the prio_msg_data, else it is a normal data message.

The second alternative is chosen such that the standard IEEE 802.15.4 is not modified. In fact, the command frame identifier which is available now $(0 \times 00)$ can be used in the future.

\subsection{Second level: the coordinator}

When receiving the message sent by the considered controller :

(1) if the controller uses a command frame to send the priority message, the command frame identifier allows the coordinator to recognize the nature of the information. This coordinator get the priority data from the first bit of the command payload.

(2) if the controller uses a command frame to send the priority message, the coordinator checks the first bit of the frame payload to identify the message. If this bit is equal to 1 , then the priority data will be set to the second bit of the frame payload.

When the coordinator receives the priority message from the controller, it broadcasts the controller priority information using a beacon frame showed in Figure 9. The first bit of the beacon payload is used to encode the controller priority information and the remaining bits are used for the controller address. The controller address will be used to identify the control loop.

\begin{tabular}{|c|c|c|c|c|c|c|c|c|}
\hline Octets:2 & 1 & 4 or 10 & 2 & variable & variable & 1 bit & variable & 2 \\
\hline $\begin{array}{c}\text { Frame } \\
\text { control }\end{array}$ & $\begin{array}{c}\text { Beacon } \\
\text { sequence } \\
\text { number }\end{array}$ & $\begin{array}{c}\text { Source } \\
\text { address } \\
\text { information }\end{array}$ & $\begin{array}{c}\text { Superframe } \\
\text { specification }\end{array}$ & $\begin{array}{c}\text { GTS } \\
\text { fields }\end{array}$ & $\begin{array}{c}\text { Pending } \\
\text { address } \\
\text { fields }\end{array}$ & Priority & $\begin{array}{c}\text { Beacon } \\
\text { payload }\end{array}$ & $\begin{array}{c}\text { Frame } \\
\text { check } \\
\text { sequence }\end{array}$ \\
\hline \multicolumn{7}{|c|}{ MAC payload } \\
MAC header & \multicolumn{7}{|l|}{ footer } \\
\hline
\end{tabular}

Fig. 9. Beacon frame format

\subsection{Third level: all nodes}

First, each node has to verify if it is the controller or the sensor of the control loop which needs better QoS. Thus the node compares the controller address to its address if they are different it compares the controller address to the destination of its data messages if they are different the node concludes that it does not belong to the considered control loop and that it has to preform the deterministic priority algorithm. The beacon_info $=1$ which means that the control loop needs better QoS, the macMinBE $E_{O A}$ is increased by 1 until it reaches its upper bound the $a M a x B E_{O A}$ and the variable which is the beginning of the range of the random delay is set to 8 to eliminate the collision with the nodes of the WNCS. Otherwise, if the beacon_info $=0$ the $\operatorname{macMinBE_{OA}}$ will be decreased by 1 until it reaches the default value which is set to 3 and the variable will be set to 0 .

\section{SIMULATION RESULTS}

TrueTime, a Matlab/Simulink simulator, is used because it allows the simulation the temporal aspects of multitasking real-time kernels and wireless networks within Simulink together with the continuous-time dynamics of the controlled plant. The online adaptive protocol with simplified priority and beacon frame is implemented and added to TrueTime package so that this protocol can be chosen as shown in Figure 10. Thus, it is on the simulated WNCS presented in Figure 2. The sensor is time-triggered whereas the controller and the actuator are event-triggered.The load generated by the two cyclops is around $90 \%$ of the total bandwidth. When using the CSMA/CA of the IEEE 802.15.4/ZigBee, the QoC of the cart is not satisfactory as shown in Figure 11.

Figure 12 presents the cart behavior when the upper bound of the error which triggers the adaptation mechanism is set to 0.5 . The QoC of the control loop is enhanced comparing 


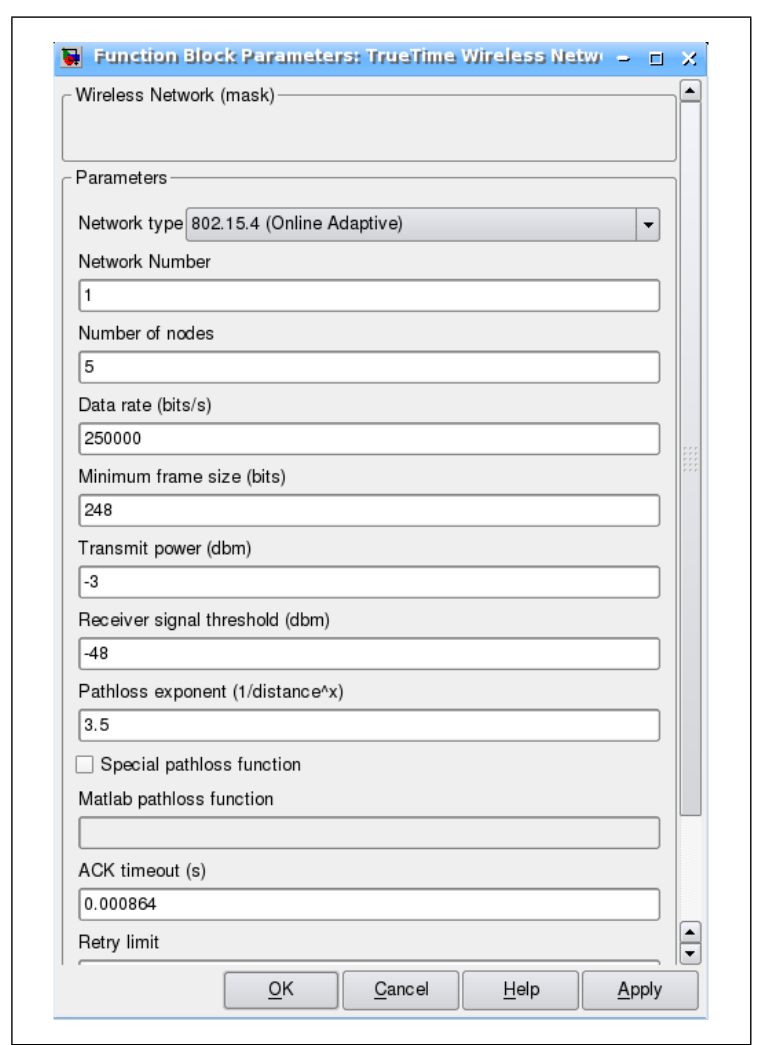

Fig. 10. TrueTime Network parameter block

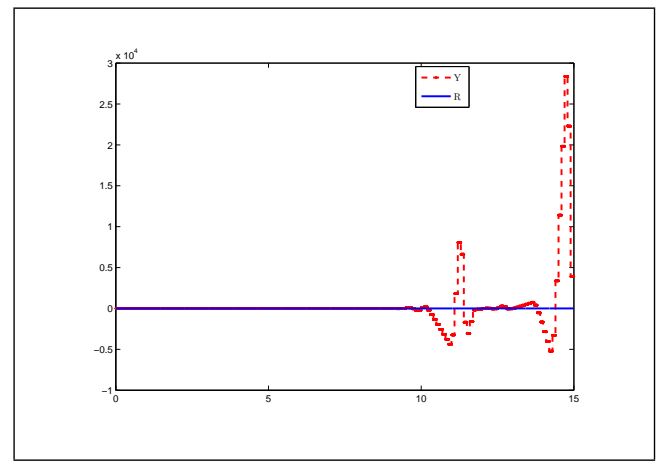

Fig. 11. The system response using CSMA/CA

to the response obtained without any QoC management as it is shown in Figure 11.

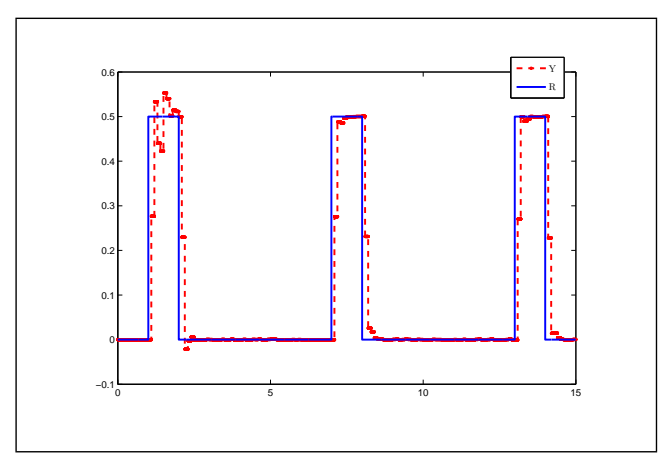

Fig. 12. The system response using the online QoC adaptive approach with threshold $=0.5$

Figure 13 and Figure 14 present the cart response for a threshold of 0.6 and respectively 0.7 . The threshold increases, the controlled system error gets more important. Thus, the time needed to bring the QoC to the desired level increases.

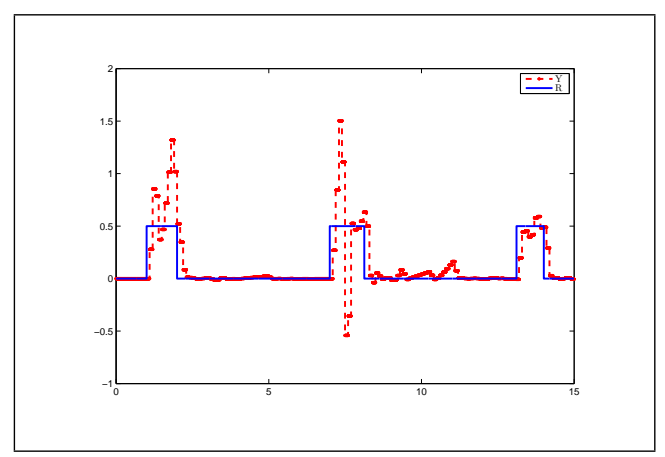

Fig. 13. The system response using the online QoC adaptive approach with threshold $=0.6$

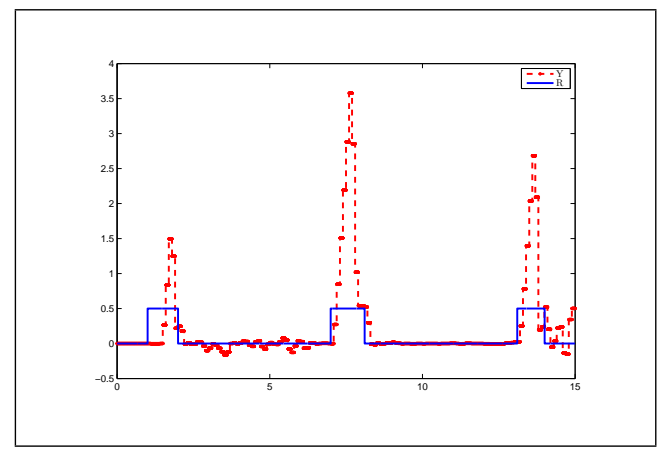

Fig. 14. The system response using the online QoC adaptive approach with threshold $=0.7$

\section{CONCLUSIONS}

In this paper, a QoS management scheme using the priority is presented. First, the case with probabilistic priority is studied and the case with deterministic priority are studied. Then, the deterministic priority QoS management scheme is adopted. An online adaptive scheme is proposed in order to manage the QoC through the adaptation of the QoS offered by the wireless network to the control loop. This QoS adaptation is ensured by means of the presented deterministic priority MAC mechanism. An implementation of this proposition is presented. This protocol is implemented and added to the TrueTime package to experience it on a simulated WNCS. The simulation results are encouraging since the QoC was enhanced with this approach. As future work, the case where there are several control loops will be studied. So, this solution must be extended to handle more than two priorities. Moreover, this online adaptive scheme will be implemented on micaz motes.

\section{REFERENCES}

[RUN] RUNES project. http://www.ist-runes.org/.

[1] HART, 2007. http://hartcomm2.org/.

[2] Karl J. Aström and Björn Wittenmark. Computercontrolled systems: theory and design (2nd ed.). Prentice-Hall, Inc., Upper Saddle River, NJ, USA, 1990. ISBN 0-13-168600-3. 
[3] N. Boughanmi, Y. Song, and E. Rondeau. Wireless networked control system using IEEE 802.15.4 with GTS. In 2nd Junior Researcher Workshop on RealTime Computing, 16-17 October 2008.

[4] F. Felicioni, N. Jia, Y. Song, and F. Simonot-Lion. Impact of a $(\mathrm{m}, \mathrm{k})$-firm data dropouts policy on the quality of control. In 6th IEEE International Workshop on Factory Communication Systems, volume Factory Communication Systems, 2006 IEEE International Workshop on, pages 353-359, Torino, Italy, 28/06/2006. http://ieeexplore.ieee.org.

[5] Kun Ji and Won jong Kim. Optimal bandwidth allocation and qos-adaptive control co-design for networked control systems. in print, International Journal of Control, Automation, and Systems, 6(4):596606 , august 2008.

[6] A. Koubâa, M. Alves, B. Nefzi, and Y. Song. Improving the ieee 802.15.4 slotted csma/ca for time-critical events in wireless sensor networks. In Workshop of Real-Time Networks (RTN 2006), Satellite Workshop to ECRTS 2006, Dresden, Germany, July 2006.

[7] P. Marti, C. Lin, S. Brandt, M. Velasco, and J. M. Fuertes. Optimal state feedback based resource allocation for resource-constrained control tasks. In 25th IEEE Real-Time Symposium (RTSS04), Lisbon, Portugal, December 2004.

[8] P. Marti, J. Yes, M. Velasco, and J.M. Fuertes. Managing quality-of-control in network-based control systems by controller and message scheduling codesign. IEEE Transactions on Industrial Electronics, 51(6), December 2004.

[9] M. Ohlin, D. Henriksson, and A. Cervin. TrueTime1.5-Reference Manual. Department of Automatic Control Lund University, 2007.

[10] V.P. Rao and D. Marandin. Adaptive backoff exponent algorithm for zigbee (IEEE 802.15.4. Next Generation Teletraffic and Wired/Wireless Advanced Networking, 4003/2006:501-516, september 2006.

[11] M. Velasco, J. M. Fuertes, C. Lin, P. Marti, and $\mathrm{S}$. Brandt. A control approach to bandwidth management in networked control systems. In 30th Annual Conference of the IEEE Industrial Electronics Society (IECON04), 2004.

[12] S. Zampieri. Trends in networked control systems. In 17th IFAC World Congress, July 6-11 2008. 